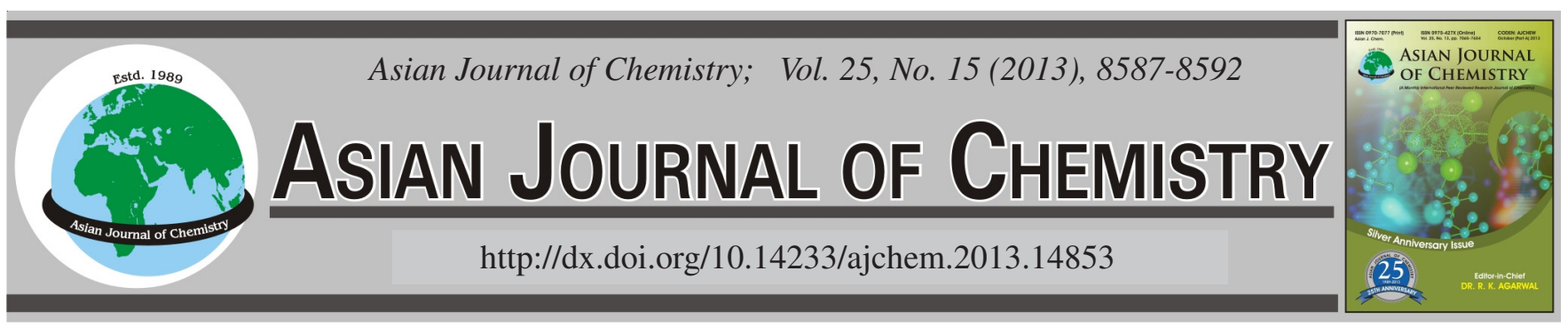

\title{
Determination on the Dissolution Rate of Doxepin Hydrochloride Tablets Produced by Different Manufacturers in China with Fiber-Optic Medicine Dissolution/Release Rate Process Test System
}

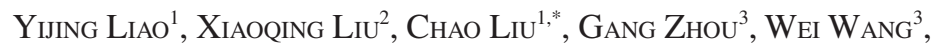 \\ Xi Mai ${ }^{1}$, Haijun Zhong ${ }^{1}$, Guggang Tu ${ }^{1}$, Huiming Huang ${ }^{1}$ and Bin Wang ${ }^{1}$
}

\begin{abstract}
${ }^{1}$ Department of Pharmacy, Medical College of Nanchang University, 461 Bayi Road, Nanchang 330006, Jiang'xi Province, P.R. China ${ }^{2}$ Jiang Xi Province Center for Disease Control and Prevention, 555 East Beijin Road, Nanchang 330029, Jiang'xi Province, P.R. China ${ }^{3}$ Mongolia Institute for Food and Drug Control, Inner Mongolia Autonomous Region 010000, P.R. China
\end{abstract}

*Corresponding author: E-mail: dxy885511@126.com

(Received: 21 November 2012;

Accepted: 26 August 2013)

AJC-14002

\begin{abstract}
The fiber-optic medicine dissolution/release rate process test system was adopted to investigate the in vitro dissolution rate curves of doxepin hydrochloride tablets on sale produced by nine different pharmaceutical factories in China. Furthermore, we compared the difference of the internal quality of these products and analyzed the controllability of quality of the existing dissolution rate method which provided effective method for drugs' quality control and the basis for establishment of dissolution rate method and standard. The paddle method was adopted, with a determination wavelength of $292 \mathrm{~nm}$, temperature $37^{\circ} \mathrm{C}$, rotating speed of $50 \mathrm{r}$ min $^{-1}$ and the time of detection was $90 \mathrm{~min}$. In different dissolution mediums, the $5 \mathrm{~mm}$ fiber-optic probe was adopted to measure the dissolution rate curves of doxepin hydrochloride tablets. Although most of the products of the nine pharmaceutical factories were found to be in accord with the regulation of Chinese Pharmacopoeia 2010. There was great difference in these dissolution curves, indicating that there were great differences in the technological level among the nine pharmaceutical factories. The analytical method used was continuous and convenient and could be used to evaluate the difference of dissolution of solid preparation.
\end{abstract}

Key Words: Doxepin hydrochloride tablet, Fiber-optic dissolution rate.

\section{INTRODUCTION}

The doxepin hydrochloride is a tricyclic antidepressant drug. It is often used to cure the anxiety melancholia and neurotic depression ${ }^{1,2}$. At present, it is the mixture of moderate amount of the cis-isomer and trans-isomer clinically.

The research on the dissolution rate was focused on determining the dissolution rate of solid preparation. This is one of the most important technological indexes on the effectiveness of solid preparation. The fiber-optic sense technology was adopted in the fiber-optic medicine dissolution/release rate process test system. It could reflect the existing issues in the preparation's technology accurately. So it could be adopted for the research on the preparation's technology and quality standard.

\section{EXPERIMENTAL}

All reagents and spectroscopic solvents were provided by Nanchang University. The adopted fiber-optic medicine dissolution/release rate process test system apparatus was FOCSDT-601. The reference standard of doxepin hydrochloride was purchased from National Institutes for Food and drug control. We collected the doxepin hydrochloride tablets on sale produced in different manufacturers in China.

Blank test: We selected three dissolution medium solutions i.e., (a) the hydrochloric acid with $\mathrm{pH} 1$; (b) the $\mathrm{pH}$ 4 buffer solution consisting of ethylic acid and natrium aceticum; (c) pH 6.8 buffer solution of phosphate. The blank test was carried out in the FOCSDT-601 with the prepared water and the three dissolution medium solutions. The results showed that the dissolution medium solutions could not disturb the determination.

Selection of detected wavelength: Briefly, $0.0157 \mathrm{mg}$ of doxepin hydrochloride reference standard was weighed accurately into a $100 \mathrm{~mL}$ measuring flask and water was added to scale. Then $50 \mathrm{~mL}$ of the solution was taken out accurately into a $250 \mathrm{~mL}$ measuring flask. It was added into the scale with water. The solution was regarded as the solution of the doxepin hydrochloride tablet dissolved completely. We also prepared the solution in the hydrochloric acid with $\mathrm{pH}$ 1.0; the $\mathrm{pH} 4.0$ buffer solution consisting of ethylic acid and natrium aceticum; pH 6.8 buffer solution of phosphate. The four solutions 

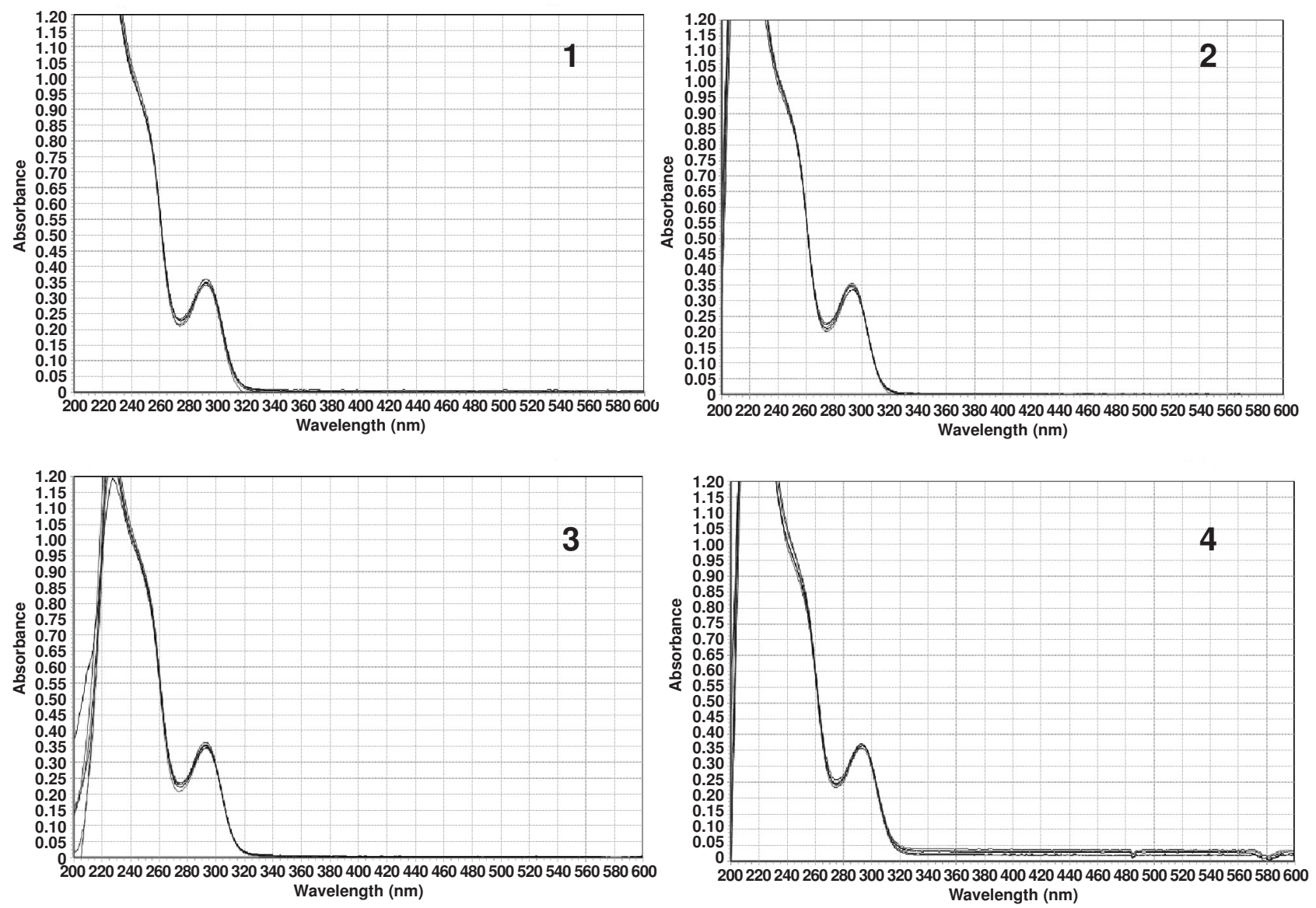

Fig. 1. Dissolution rate of doxepin hydrochloride reference standard in different dissolution mediums ( 1 was in water; 2 was hydrochloric acid with pH 1 ; 3 was the $\mathrm{pH} 4$ buffer solution consisting of ethylic acid and natrium aceticum; 4 was $\mathrm{pH} 6.8$ buffer solution of phosphate)

were tested in the FOCSDT-601. The specification of the probe was $5 \mathrm{~mm}$ and the volume of solvent was $900 \mathrm{~mL}$. The solution with water was dissolved for $45 \mathrm{~min}$, while the other three solutions were dissolved for $90 \mathrm{~min}$. From the Fig. 1, the maximum wavelength was $292 \mathrm{~nm}$. It was confirmed as the detected wavelength. The reference wavelength was $55 \mathrm{~nm}$.

Selection of apparatus and rotation rate: The stirring basket method was adopted. The rotation rate was $100 \mathrm{rpm}$. The same batches of doxepin hydrochloride tablets produced by the same pharmaceutical factory were dissolved in the four different dissolution mediums earlier mentioned ${ }^{3,4}$. The results were shown in the Fig. 2.

Linear relation: The moderate amount of doxepin hydrochloride reference standard was taken out. After drying at $105^{\circ} \mathrm{C}$ for $4 \mathrm{~h}, 30.15 \mathrm{mg}$ of doxepin hydrochloride reference standard was weighed accurately into a $100 \mathrm{~mL}$ measuring flask. It was added to the scale with de-aerated water and was regarded as the stock solution. Next, 2, 4, 6, 8, 10 and $12 \mathrm{~mL}$ of the stock solution were put into $100 \mathrm{~mL}$ measuring flasks. They were added to the scale with de-aerated water to a concentration of 19.4, 38.8, 58.2, 77.6, 97.0 and $116.4 \%$, respectively. These prepared solutions were analyzed in FOCSDT-601. The results are shown at Table-1 and Fig. 3.

\section{RESULTS AND DISCUSSION}

Methodological verification In present experiment of selecting apparatus and rotation rate, we found out that the

\begin{tabular}{ccccccc}
\multicolumn{7}{c}{ TABLE-1 } \\
\multicolumn{7}{c}{ OBTAINED DATA OF EXPERIMENT } \\
ON LINEAR RELATIONSHIP \\
\hline $\mathrm{X}$ & 19.4 & 38.8 & 58.2 & 77.6 & 97.0 & 116.4 \\
\hline $\mathrm{Y}$ & 0.064563 & 0.131883 & 0.203893 & 0.282071 & 0.340434 & 0.40923 \\
\hline
\end{tabular}

obtained result of dissolution was much higher than the actual result because accessories were deposited in the groove of probe of fiber in the procession of dissolution. Hence the paddle method was adopted. The rotation rate was adjusted as $50 \mathrm{rpm}$. The same batches of doxepin hydrochloride tablets produced by the same pharmaceutical factory were dissolved in the four different dissolution mediums earlier mentioned and the results were shown in the Fig. 4. And there was a favorable linear relationship between the concentrations of doxepin hydrochloride and absorbance in the range of $0-120 \%$ of concentrations of doxepin hydrochloride. In addition, when the results with fibre and results with UV method were compared, RSD was $1.2 \%$. This showed that there was no obvious difference between the results with fibre and results with UV method.

Determination on the doxepin hydrochloride tablets on sale in China: Using the analytical method aforementioned, we analyzed the stripping curve of doxepin hydrochloride tablets on sale produced by nine different pharmaceutical factories in China. We adopted the $\mathrm{f} 2$ gene method on evaluating the comparability of stripping curve of doxepin hydrochloride tablets on sale in China. 
2
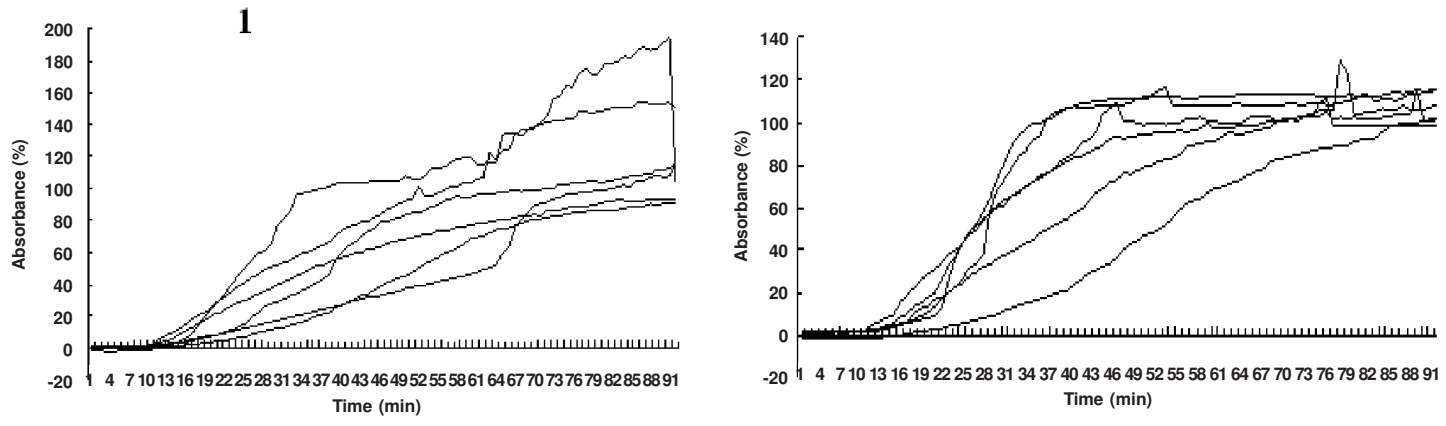

3

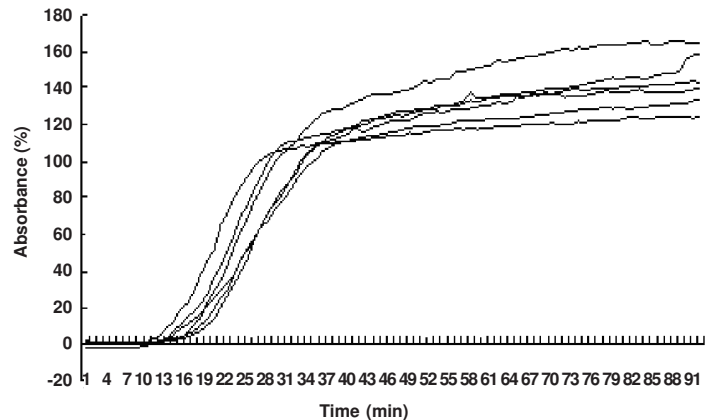

4

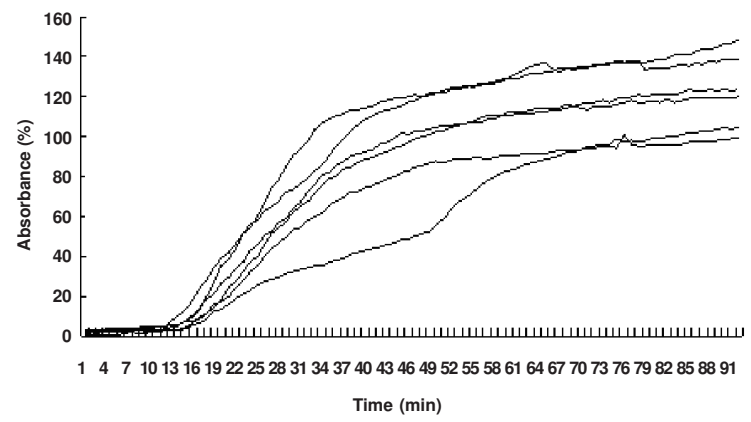

Fig. 2. Stripping curves of same batches of doxepin hydrochloride tablets in different dissolution mediums (1 was in water; 2 was hydrochloric acid with $\mathrm{pH}$ $1.0 ; 3$ was the $\mathrm{pH} 4.0$ buffer solution consisting of ethylic acid and natrium aceticum; 4 was $\mathrm{pH} 6.8$ buffer solution of phosphate)

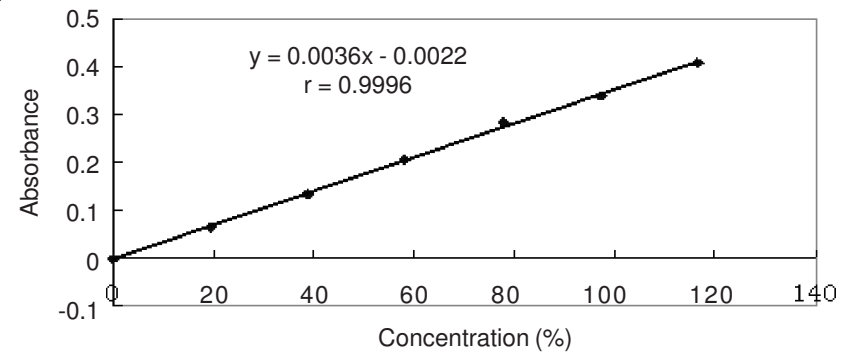

Fig. 3. Linear curve of doxepin hydrochloride

1

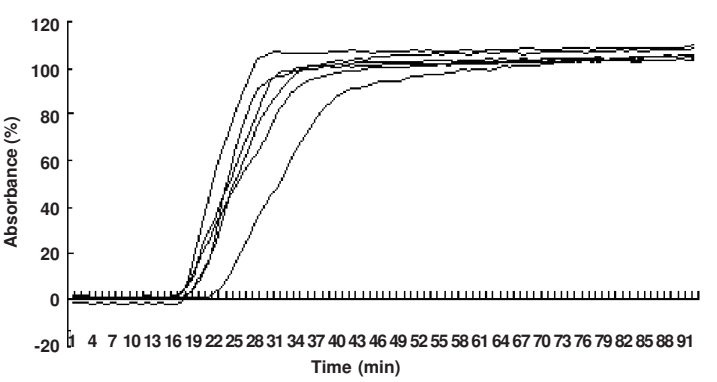

3

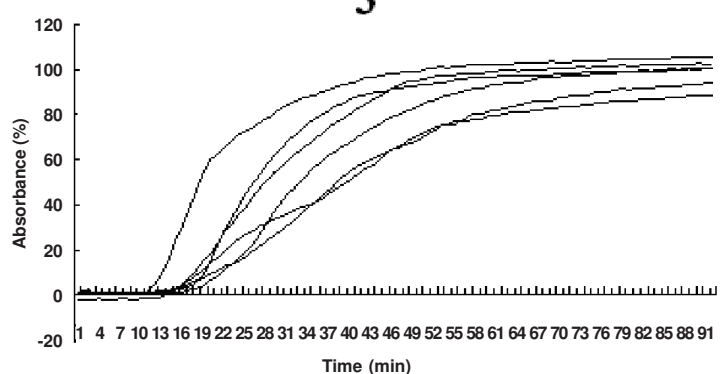

Comparison of stripping curve of the doxepin hydrochloride tablets produced by the same pharmaceutical factory in different dissolution mediums: We determined the stripping curve of the doxepin hydrochloride tablet produced by the same pharmaceutical factory in different dissolution mediums. The tablets were collected from nine pharmaceutical factories. The results are shown at Tables 210 and Fig. 5. Besides, we named the nine different pharmaceutical factories as factory $1,2,3,4,5,6,7,8$ and 9 .

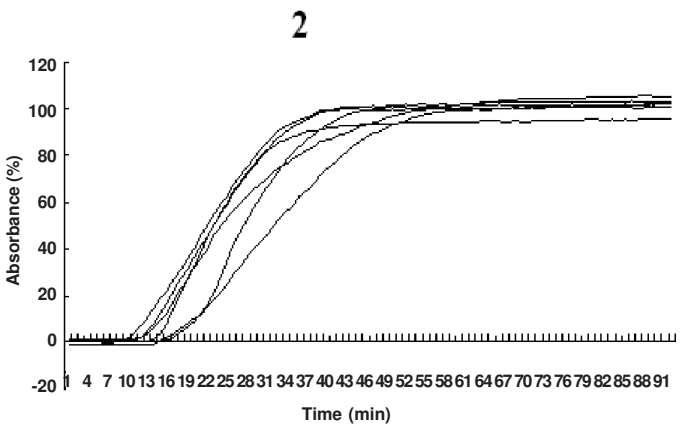

4

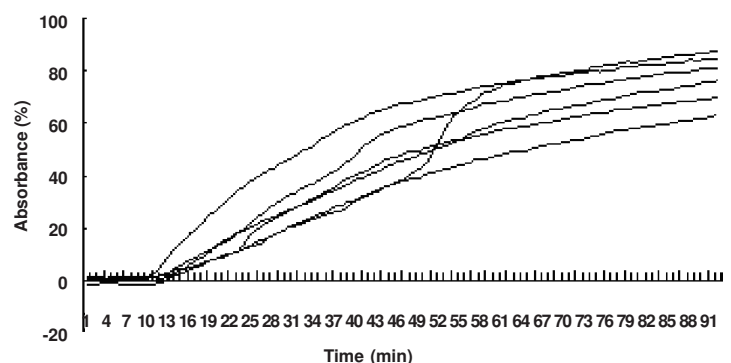

Fig. 4. Results obtained by the paddle method ( 1 was in water; 2 was hydrochloric acid with $\mathrm{pH} 1.0 ; 3$ was the $\mathrm{pH} 4.0$ buffer solution consisting of ethylic acid and natrium aceticum; 4 was $\mathrm{pH} 6.8$ buffer solution of phosphate) 


\begin{tabular}{cccccc}
\hline \multicolumn{6}{c}{ TABLE-2 } \\
$\begin{array}{c}\text { DETERMINATION ON THE DISSOLUTION OF DOXEPIN } \\
\text { HYDROCHLORIDE TABLET FROM FACTORY 1 }\end{array}$ \\
\hline Reference & pH 1 & f2 & Conclusion & AV & Conclusion \\
\hline Tested & water & -751.1 & Dissimilar & 53.6 & Dissimilar \\
Tested & pH 4 & -751.1 & Dissimilar & 60.0 & Dissimilar \\
Tested & pH 6.8 & -748.6 & Dissimilar & 41.7 & Dissimilar \\
\hline
\end{tabular}

TABLE-3

DETERMINATION ON THE DISSOLUTION OF DOXEPIN HYDROCHLORIDE TABLET FROM FACTORY 2

\begin{tabular}{cccccc}
\hline Reference & pH 4 & f2 & Conclusion & AV & Conclusion \\
\hline Tested & water & 76.7 & similar & 36.6 & Dissimilar \\
Tested & pH 1 & 76.7 & similar & 37.7 & Dissimilar \\
Tested & pH 6.8 & 47.6 & dissimilar & 54.4 & Dissimilar \\
\hline
\end{tabular}

\begin{tabular}{|cccccc}
\multicolumn{8}{c}{ TABLE-4 } \\
\multicolumn{7}{|c}{ DETERMINATION ON THE DISSOLUTION OF DOXEPIN } \\
HYDROCHLORIDE TABLET FROM FACTORY 3 \\
\hline Reference & water & f2 & Conclusion & AV & Conclusion \\
\hline Tested & pH 1 & - & - & 38.5 & Dissimilar \\
Tested & pH 4 & - & - & 49.6 & Dissimilar \\
Tested & pH 6.8 & - & - & 35.8 & Dissimilar \\
\hline
\end{tabular}

\begin{tabular}{|cccccc}
\hline \multicolumn{7}{c}{ TABLE-5 } \\
DETERMINATION ON THE DISSOLUTION OF DOXEPIN \\
HYDROCHLORIDE TABLET FROM FACTORY 4 \\
\hline Reference & $\mathrm{pH} 4$ & $\mathrm{f} 2$ & Conclusion & AV & Conclusion \\
\hline Tested & Water & 73.1 & Similar & 62.7 & Dissimilar \\
Tested & $\mathrm{pH} \mathrm{1}$ & 61.7 & Similar & 84.6 & Dissimilar \\
Tested & $\mathrm{pH} 6.8$ & 58.3 & Similar & 50.3 & Dissimilar \\
\hline
\end{tabular}

TABLE-6

DETERMINATION ON THE DISSOLUTION OF DOXEPIN HYDROCHLORIDE TABLET FROM FACTORY 5

\begin{tabular}{cccccc}
\hline Reference & pH 1 & f2 & Conclusion & AV & Conclusion \\
\hline Tested & Water & - & - & 62.9 & Dissimilar \\
Tested & pH 4 & 76.3 & Similar & 55.7 & Dissimilar \\
Tested & pH 6.8 & - & - & 62.8 & Dissimilar \\
\hline
\end{tabular}

TABLE-7

DETERMINATION ON THE DISSOLUTION OF DOXEPIN HYDROCHLORIDE TABLET FROM FACTORY 6

\begin{tabular}{cccccc}
\hline Reference & water & f2 & Conclusion & AV & Conclusion \\
\hline Tested & pH 1 & 26.6 & Dissimilar & 69.4 & Dissimilar \\
Tested & pH 4 & 26.8 & Dissimilar & 77.5 & Dissimilar \\
Tested & pH 6.8 & 26.4 & Dissimilar & 90.0 & Dissimilar \\
\hline
\end{tabular}

TABLE-8

DETERMINATION ON THE DISSOLUTION OF DOXEPIN HYDROCHLORIDE TABLET FROM FACTORY 7

\begin{tabular}{cccccc}
\hline Reference & pH4 & f2 & Conclusion & AV & Conclusion \\
\hline Tested & Water & 75.7 & Similar & 58.7 & Dissimilar \\
Tested & pH 1 & 78.2 & Similar & 60.4 & Dissimilar \\
Tested & pH 6.8 & 68.0 & Similar & 52.0 & Dissimilar \\
\hline
\end{tabular}

TABLE-9

DETERMINATION ON THE DISSOLUTION OF DOXEPIN HYDROCHLORIDE TABLET FROM FACTORY 8

\begin{tabular}{cccccc}
\hline Reference & Water & f2 & Conclusion & AV & Conclusion \\
\hline Tested & pH 1 & 72.2 & Similar & 41.6 & Dissimilar \\
Tested & pH 4 & 87.4 & Similar & 36.0 & Dissimilar \\
Tested & pH6.8 & 92.5 & Similar & 38.1 & Dissimilar \\
\hline
\end{tabular}

\begin{tabular}{cccccc}
\hline \multicolumn{6}{c}{ TABLE-10 } \\
\multicolumn{6}{c}{ DETERMINATION ON THE DISSOLUTION OF DOXEPIN } \\
HYDROCHLORIDE TABLET FROM FACTORY 9 \\
\hline Reference & Water & f2 & Conclusion & AV & Conclusion \\
\hline Tested & pH 1 & 52.7 & Similar & 58.4 & Dissimilar \\
Tested & pH 4 & 51.3 & Similar & 61.1 & Dissimilar \\
Tested & pH6.8 & 48.1 & Dissimilar & 81.6 & Dissimilar \\
\hline
\end{tabular}
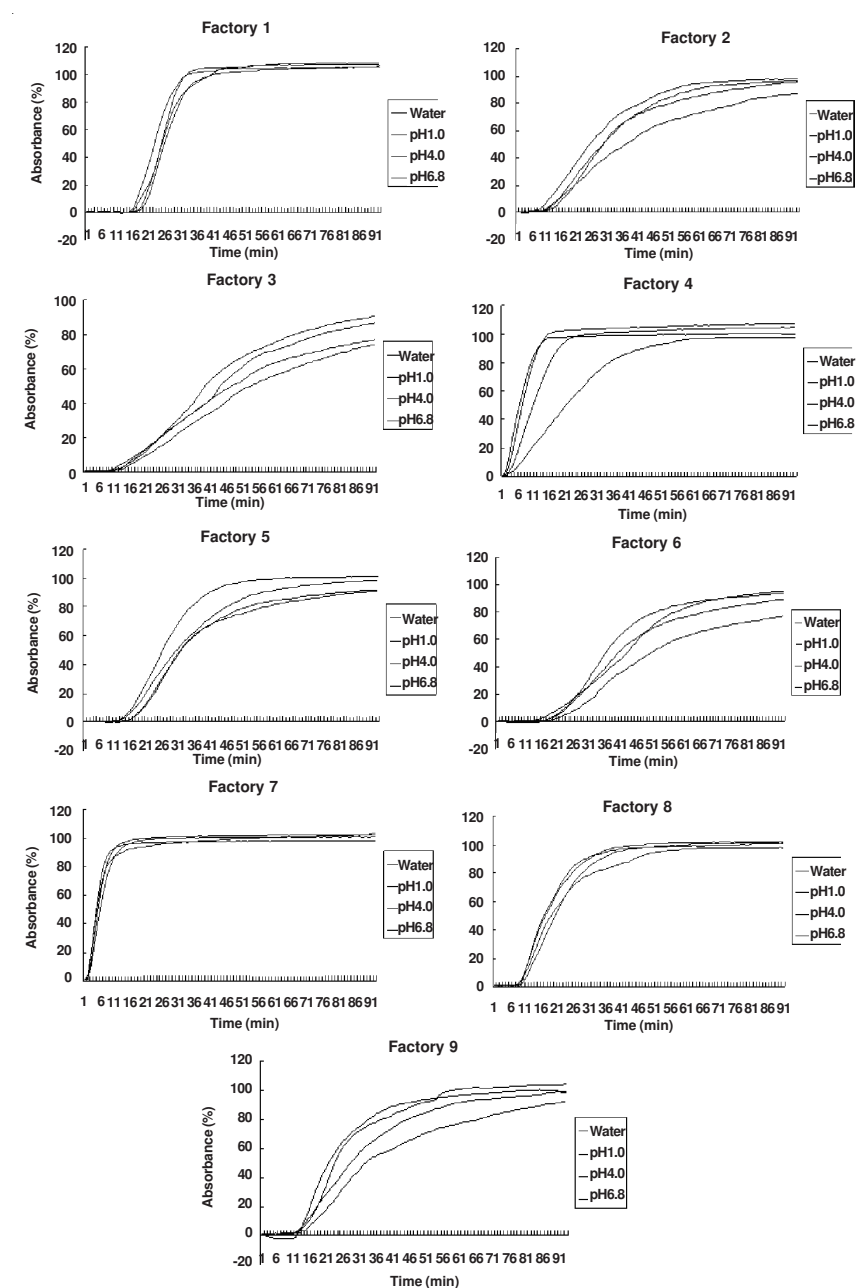

Fig. 5. Stripping curve of the doxepin hydrochloride tablet produced by the different pharmaceutical factories in different dissolution medium

Comparison of stripping curve of the doxepin hydrochloride tablets produced by different pharmaceutical factories in the same dissolution medium: From the results obtained above, we found out that there was little difference between the doxepin hydrochloride tablets produced by factory 4 and tablet produced by factory 7 . Besides, their stripping curves were representative. Hence, the hydrochloride tablets produced by factory 4 and factory 7 were selected as reference preparations. The hydrochloride tablets produced by the other factories were regarded as tested preparations. The results were shown in Tables 11-14 and Figs. 6-9.

\section{Conclusion}

From the comparison of stripping curves, we found out that stripping curve of doxepin hydrochloride tablets produced by factory 7 was the closest to the standard stripping curve and it could be released in different dissolution mediums 
TABLE-11

COMPARISON OF STRIPPING CURVE IN WATER

\begin{tabular}{cccccc}
\hline Reference & Factory 4 & f2 & Conclusion & AV & Conclusion \\
\hline Tested & Factory 1 & 74.3 & Similar & 111.4 & Dissimilar \\
Tested & Factory 2 & 58.1 & Similar & 108.1 & Dissimilar \\
Tested & Factory 3 & 46.2 & Dissimilar & 109.3 & Dissimilar \\
Tested & Factory 5 & 76.2 & Similar & 63.1 & Dissimilar \\
Tested & Factory 6 & - & - & 111.3 & Dissimilar \\
Tested & Factory 7 & - & - & 111.6 & Dissimilar \\
Tested & Factory 8 & 90.8 & Similar & 105.4 & Dissimilar \\
Tested & Factory 9 & 63.4 & Similar & 108.5 & Dissimilar \\
\hline Reference & Factory 7 & f2 & Conclusion & AV & Conclusion \\
\hline Tested & Factory 1 & 94.7 & Similar & 60.6 & Dissimilar \\
Tested & Factory 2 & 51.8 & Similar & 61.9 & Dissimilar \\
Tested & Factory 3 & 42.3 & Dissimilar & 68.0 & Dissimilar \\
Tested & Factory 4 & - & - & 70.9 & Dissimilar \\
Tested & Factory 5 & - & - & 71.3 & Dissimilar \\
Tested & Factory 6 & 72.6 & Similar & 53.3 & Dissimilar \\
Tested & Factory 8 & 69.5 & Similar & 54.7 & Dissimilar \\
Tested & Factory 9 & 55.0 & Similar & 59.4 & Dissimilar \\
\hline
\end{tabular}

TABLE-12

COMPARISON OF STRIPPING CURVE IN pH 1.0 HYDROCHLORIC ACID

\begin{tabular}{cccccc}
\hline Reference & Factory 4 & f2 & Conclusion & AV & Conclusion \\
\hline Tested & Factory 1 & 72.8 & Similar & 98.7 & Dissimilar \\
Tested & Factory 2 & 55.8 & Similar & 95.5 & Dissimilar \\
Tested & Factory 3 & - & - & 95.5 & Dissimilar \\
Tested & Factory 5 & 58.1 & Similar & 84.6 & Dissimilar \\
Tested & Factory 6 & 75.2 & Similar & 97.4 & Dissimilar \\
Tested & Factory 7 & 91.4 & Similar & 98.1 & Dissimilar \\
Tested & Factory 8 & 68.5 & Similar & 90.7 & Dissimilar \\
Tested & Factory 9 & 59.7 & Similar & 94.5 & Dissimilar \\
\hline Reference & Factory 7 & f2 & Conclusion & AV & Conclusion \\
\hline Tested & Factory 1 & 49.2 & Dissimilar & 45.8 & Dissimilar \\
Tested & Factory 2 & 72.0 & Similar & 46.7 & Dissimilar \\
Tested & Factory 3 & - & - & 60.9 & Dissimilar \\
Tested & Factory 4 & 68.9 & Similar & 39.3 & Dissimilar \\
Tested & Factory 5 & 87.0 & Similar & 57.6 & \\
Tested & Factory 6 & 59.1 & Similar & 65.7 & Dissimilar \\
Tested & Factory 8 & 97.0 & Similar & 37.2 & Dissimilar \\
Tested & Factory 9 & 72.8 & Similar & 42.0 & Dissimilar \\
\hline
\end{tabular}

TABLE-13

COMPARISON OF STRIPPING CURVE IN pH 4.0 HYDROCHLORIC ACID

\begin{tabular}{cccccc}
\hline Reference & Factory 4 & f2 & Conclusion & AV & Conclusion \\
\hline Tested & Factory 1 & 51.6 & Similar & 99.0 & dissimilar \\
Tested & Factory 2 & 57.5 & Similar & 98.1 & dissimilar \\
Tested & Factory 3 & - & - & 98.9 & dissimilar \\
Tested & Factory 5 & 84.9 & Similar & 38.4 & dissimilar \\
Tested & Factory 6 & 100.0 & Similar & 99.0 & dissimilar \\
Tested & Factory 7 & - & - & 99.1 & dissimilar \\
Tested & Factory 8 & 74.9 & Similar & 97.3 & dissimilar \\
Tested & Factory 9 & 64.8 & Similar & 97.8 & dissimilar \\
\hline Reference & Factory 7 & f2 & Conclusion & AV & Conclusion \\
\hline Tested & Factory 1 & 56.4 & Similar & 89.3 & dissimilar \\
Tested & Factory 2 & 53.9 & Similar & 88.1 & dissimilar \\
Tested & Factory 3 & - & - & 88.7 & dissimilar \\
Tested & Factory 4 & 83.9 & Similar & 90.9 & dissimilar \\
Tested & Factory 5 & - & - & 89.3 & dissimilar \\
Tested & Factory 6 & 84.9 & Similar & 30.1 & dissimilar \\
Tested & Factory 8 & 89.8 & Similar & 79.9 & dissimilar \\
Tested & Factory 9 & 73.7 & Similar & 86.8 & dissimilar \\
\hline
\end{tabular}

TABLE-14

COMPARISON OF STRIPPING CURVE IN pH 6.8 BUFFER SOLUTION OF PHOSPHATE

\begin{tabular}{cccccc}
\hline Reference & Factory 4 & f2 & Conclusion & AV & Conclusion \\
\hline Tested & Factory 1 & 18.6 & dissimilar & 95.9 & dissimilar \\
Tested & Factory 2 & 39.8 & dissimilar & 95.8 & dissimilar \\
Tested & Factory 3 & - & - & 95.3 & dissimilar \\
Tested & Factory 5 & 63.6 & similar & 36.1 & dissimilar \\
Tested & Factory 6 & - & - & 95.4 & dissimilar \\
Tested & Factory 7 & 1.2 & dissimilar & 96.4 & dissimilar \\
Tested & Factory 8 & 71.3 & similar & 93.1 & dissimilar \\
Tested & Factory 9 & - & - & 95.8 & dissimilar \\
\hline Reference & Factory 7 & f2 & Conclusion & AV & Conclusion \\
\hline Tested & Factory 1 & 37.7 & dissimilar & 98.5 & dissimilar \\
Tested & Factory 2 & 25.4 & dissimilar & 94.1 & dissimilar \\
Tested & Factory 3 & - & - & 96.4 & dissimilar \\
Tested & Factory 4 & - & - & 98.0 & dissimilar \\
Tested & Factory 5 & 20.3 & dissimilar & 99.5 & dissimilar \\
Tested & Factory 6 & 71.8 & similar & 57.2 & dissimilar \\
Tested & Factory 8 & 56.6 & similar & 81.7 & dissimilar \\
Tested & Factory 9 & 20.5 & dissimilar & 96.9 & dissimilar \\
\hline
\end{tabular}

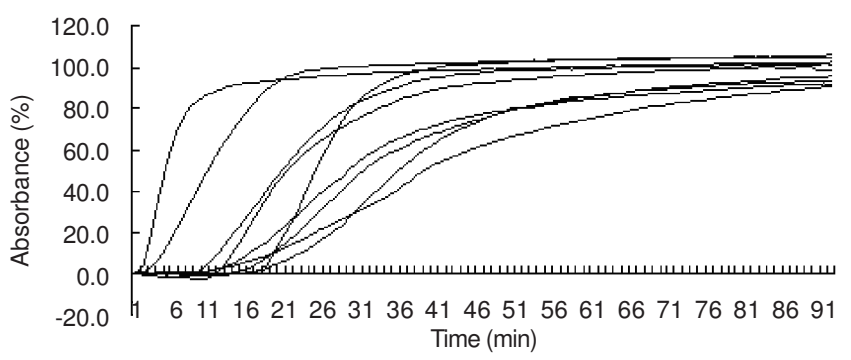

Fig. 6. Comparison of stripping curve in water

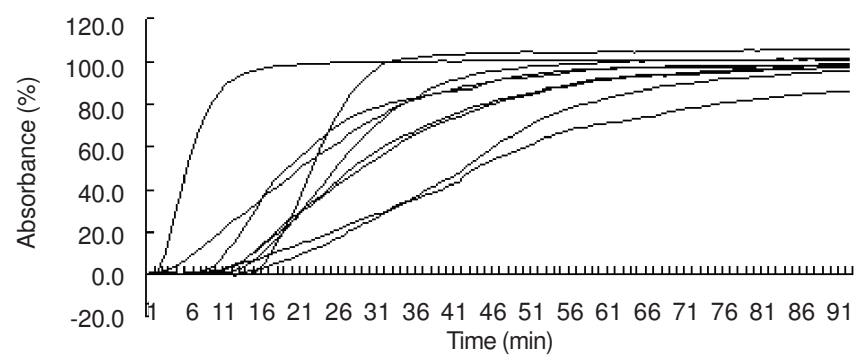

Fig. 7. Comparison of stripping curve in $\mathrm{pH} 1$ hydrochloric acid

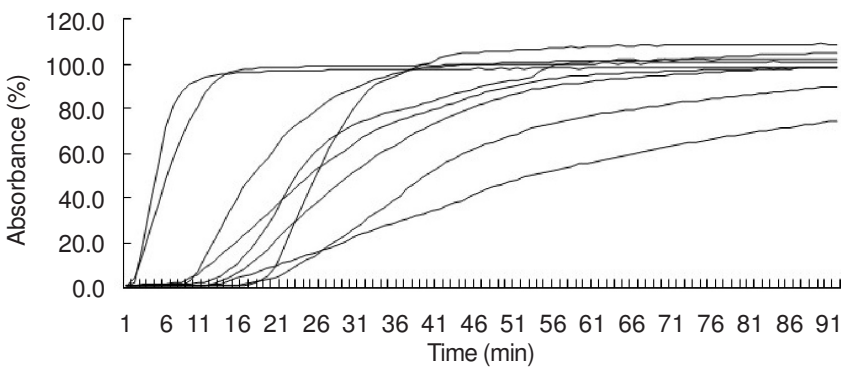

Fig. 8. Comparison of stripping curve in $\mathrm{pH} 4$ hydrochloric acid

equably and well. This indicated that it could be adopted on patients with different body constitution well. The second closest to the standard stripping curve was the stripping curve of doxepin hydrochloride tablets produced by factory 4 . The dosage form of the two factories was film coated tablet, while the dosage form of the other factories was sugar coated tablet. So we found out the film coated tablet was much better than 


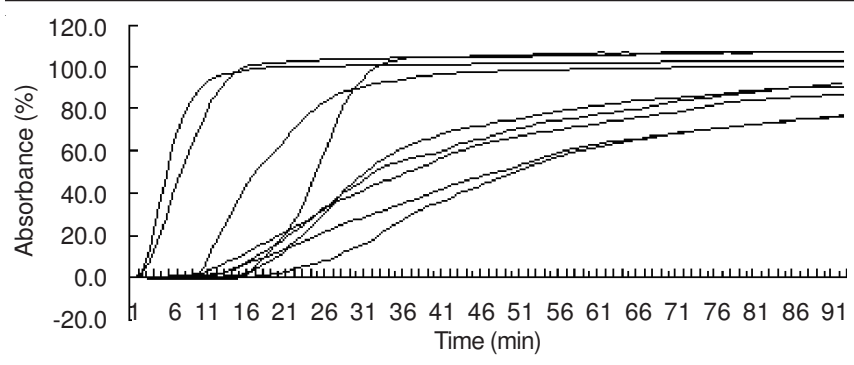

Fig. 9. Comparison of stripping curve in $\mathrm{pH} 6.8$ buffer solution of phosphate

traditional dosage form as sugar coated tablet. Our results therefore established an exoteric and accurate analytical method on evaluating inherent quality of doxepin hydrochloride tablets.

\section{ACKNOWLEDGEMENTS}

The financial support from the program of Department of Health of JiangXi Province(NO. 20112014) is gratefully acknowledged.

\section{REFERENCES}

1. M.E. Széliga, M.C. Lamas, D.A. Lillo and C. Bregni, Boll. Chim. Farm., 136, 527 (1997).

2. US Department of Health and Human Services Food and Drug Administration Center for Drug Evaluation and Research (CDER) Guidance for Industry Dissolution Testing of Immediate Release Solid Oral Dosage Forms (1997).

3. C. Gido, P. Langguth and E. Mutschler, Pharm. Res., 11, 800 (1994).

4. C. Gido, P. Langguth, J. Kreuter, G. Winter, H. Woog and E. Mutschler, Pharmazie, 48, 764 (1993). 